\title{
Psicología, pedagogía e historia: los caminos que ofrecen los Estudios Sociales de la Ciencia para el análisis de la movilización de dispositivos conceptuales y prácticos ${ }^{1}$
}

\section{Psychology, education and history: the paths offered by social studies of science to analyze the mobilization of conceptual and practice devices}

\author{
Joan Sebastián Soto Triana ${ }^{2}$ \\ Universidad Nacional de Colombia, Bogotá, Colombia
}

(Rec: marzo 2015 - Acept: junio 2015)

\begin{abstract}
Resumen
El presente escrito proporciona una reflexión sobre la manera en que se ha constituido el análisis de la historia de la psicología en Colombia. Aporta un desarrollo conceptual a la tradición clásica de ver la historia como una mención a momentos y personajes "heroicos" descuidando posibilidades analíticas alrededor de diversas narraciones que posibilitan una comprensión amplia de los movimientos de la psicología como espacio de apropiación social del conocimiento, construcción de redes sociotécnicas, prácticas de traducción de intereses. A través de la breve exposición del caso de la psicología y la pedagogía en el centro educativo Gimnasio Moderno de Bogotá en los inicios del siglo XX se observan como los Estudios Sociales de la Ciencia proveen herramientas importantes en función de su epistemología y metodología para hacer seguimiento de los conceptos, las prácticas, las adaptaciones y puestas en escena de la psicología del desarrollo europea en un plantel educativo donde la infancia se torna "paso obligado" de los discursos sobre modernización y moral.

Palabras clave: historia de la psicología, estudios sociales de la ciencia y psicología, psicología y pedagogía.
\end{abstract}

\begin{abstract}
This paper provides a reflection about the way in which the analysis of the history of psychology in Colombia has been constituted. It contributes a conceptual development to the classical tradition of viewing history as a reference to moments and "heroic" characters, neglecting analytical possibilities around various narratives that enable a broad understanding of the movements of psychology as a space for social appropriation of knowledge, sociotechnical network building and practices of translation of interests. Through a brief exposition of the case of psychology and education at the Gimnasio Moderno School of Bogota in the early twentieth century, the way in which Social Studies of Science provide important tools in terms of their epistemology and methodology for monitoring concepts, practices, adaptations and staging of European developmental psychology in an educational institution where childhood is a "mandatory step" in narratives about modernization is presented.

Keywords: history of psychology, social studies of science, psychology and education.
\end{abstract}

\footnotetext{
1 Este artículo está asociado al proyecto de investigación "Relaciones Internacionales Tecnocientíficas de América Latina con Alemania y Estados Unidos Durante el S.XX”, código 201010016521. Unidad Camilo Torres. Calle. 44 No. 45-67 - Bloque B5. 3165000 ext. 10435, 10436.

2 Correspondencia a: Joan Sebastian Soto Triana. Corporación Universitaria Minuto de Dios, Programa de Psicología, Calle 81B \# 72B - 70, Ciudad de Bogotá, Colombia. Tel: (+57 1) 5933004. E-mail: joasototria@gmail.com.
} 


\section{Introducción}

La psicología en Colombia, como en otros países de Latinoamérica, ha tenido desarrollos variados en términos de sistemas epistemológicos, conceptuales y metodológicos que han generado una variedad de perspectivas afiliadas a personas, instituciones e intereses. Como ha sugerido Klappenbach y Pavessi (1994), en su ya clásico trabajo sobre las perspectivas de la historia de la psicología en Latinoamérica, es difícil construir un relato originario de la manera en que los conceptos psicológicos comienzan a andar sobre las ruedas de la ciencia para consolidar una perspectiva de trabajo y disciplina profesional.

Una perspectiva de trabajo ya clásica en la historia de la psicología en Colombia, que ha sido heredada de los textos de Ardila (1973, 1999, 2004), sugiere que las actividades científicas en América Latina estuvieron precedidas por un interés originario de reproducir una forma de trabajo empírico derivada de las aproximaciones a las grandes influencias de la psicología europea y norteamericana. Es interesante también detallar que a esta tradición historiográfica, se han unido bastantes historias de la psicología en Colombia que ha acogido la tradición de Ardila como un referente importante para rescatar cierto canon y un relato coherente que concentra la atención en la historia de la ciencia como producto del quehacer de personajes que de manera "heroica" han logrado consolidar un campo de investigación y aplicación (Jaraba, 2012; Mora, 2012).

Ahora bien, esta tradición que se ha rescatado en la historia de la psicología colombiana, tiene un componente importante en su haber; la referencia constante de Ardila $(1973,1975)$ y sus seguidores (Ardila \& Pérez-Acosta, 2010; Gutiérrez, Pérez-Acosta \& Plata-Caviedes, 2009; López, 1997; Mankeliunas, 1980, 1993; Peña, 1993; Pérez-Acosta, 1997) a la importancia de la afiliación a un método de trabajo basado en la experimentación y la posibilidad de generar una perspectiva de trabajo empírico que diera cimientos a la ciencia del comportamiento como lugar de la validez y objetividad del conocimiento. En este sentido, la perspectiva de trabajo que se privilegia en la narrativa histórica es la aparición del trabajo empírico como evidencia de un crecimiento en pos del desarrollo de la psicología como ciencia, independiente de sus filiaciones con la medicina, la filosofía y la pedagogía.

De este modo, la psicología en el canon de la historia ya referenciada, se encuentra del lado de una perspectiva empírico analítica, al parecer por un sentido de vocación de los "pioneros" que se vieron interesados por el desarrollo de una perspectiva científica. Esta incluía, de manera sustancial, la referencia a la estadística como conteo de las características individuales, susceptibles a la comparación con un perfil. Por otro lado, la metodología experimental se inscribe como el pilar de la proyección del escenario de producción de conocimiento ante otras comunidades de mayor trayectoria en América Latina, Estados Unidos y Europa.

La tradición del canon de la psicología en los estudios históricos ha invisibilizado autores que han tratado de generar un proceso de investigación de narraciones al margen de aquellas que se han presentado. Está el caso de las posibilidades que han surgido de las revisiones historiográficas de Oviedo $(2009,2013)$, con su interesante desarrollo de la perspectiva psicológica de finales del siglo XIX, asociada a tradiciones neotomistas en la religión y que tienen una influencia poderosa en la consolidación de un proyecto de religiosidad y pedagogía a través de la política "regeneracionista" en el país. El desarrollo de las perspectivas teóricas asociadas a la psicología de la Gestalt y la interesante vuelta al reconocimiento de Manuel Ancízar, como estudioso y desarrollador de conceptos psicológicos ligados al concepto de conciencia (Oviedo, 2004, 2010).

Ahora bien, tenemos los interesantes avances de la psicología ligada a la perspectiva de la "Escuela Activa o Nueva" que se dio en el seno de los 30 primeros años del siglo XX en el país. Esta iniciativa estuvo a cargo de algunas instituciones educativas que recogen ideas y presupuestos psicológicos de la escuela del desarrollo de María Montessori y Ovide Decroly, pasando por la interpretación de ideas importantes de la pedagogía activa, propuesta por estos autores con la influencia de perspectivas teóricas de la Escuela de Ginebra (Sáenz, 1990; Sáenz \& Zuluaga, 2004; Sáenz, Saldarriaga \& Ospina, 1997).

Otros interesantes trabajos derivados de propuestas historiográficas renovadoras, son los que en los últimos años nos han ofrecido los Estudios Sociales de la Ciencia y la Tecnología (ESCT en adelante), reconociendo a la psicología como dispositivo tecnocientífico. Se pueden observar complejos tipos de redes elaborados por Jaraba (2012) que buscan dar una comprensión a la institución de la psicotecnia como experticia que legitima intereses políticos en la conformación de los exámenes de ingreso a la Universidad Nacional, teniendo a Mercedes Rodrigo como un actor relevante que traduce intereses desde la misma disciplina con relación a motivos de sectores políticos y sociales de la época en el país. Encontramos también el clarificador trabajo de Mora (2012) sobre la red de 
movilización de la terapia conductual en diferentes centros de enseñanza, y la utilización de este aparataje tecnocientífico como instrumento de resocialización, en torno a la controversia de los usos de una técnica de "economía de fichas" en la cárcel del "Buen Pastor" en Bogotá en los años setenta.

En la siguiente sección se hablará sobre las últimas posibilidades analíticas mencionadas. Concretamente, el foco estará en la posible relación metodológica que brinda los ESCT en el trabajo de generar narraciones sobre la historia de la psicología; no como una historia de héroes y épica, sino aquella que permita un análisis de sus redes, movilización, intereses, motivaciones y producción de instituciones, usos, socialización y cultura.

\section{Los Estudios Sociales de la Ciencia y la Tecnología (ESCT) y la posibilidad de análisis de la historia de la psicología y la pedagogía}

Una de las más interesantes situaciones que se observa en la historiografía de la psicología en el país, es la relación que existe entre narraciones y cánones, e incluso de los estilos de presentación del relato y la información (Jaraba, 2012; Mora, 2012). Como se ha descrito más arriba, la función social percibida de esta presentación de la historia, obedece a formas institucionales destinadas a fortalecer perspectivas particulares de la concepción de los hechos de la psicología. Se observa entonces como la psicología en sus inicios y desarrollos se cuenta como historia de la ciencia y como historia de un ethos particularmente "heroico" de los hechos de movilización de conceptos y tecnologías que aportan, desde "centros" especializados, las fuentes de desarrollo de un país del tercer mundo.

Cuando se observan las tradiciones historiográficas referidas a la posibilidad de comprender los fenómenos de movilización de conceptos científicos, técnicos y tecnológicos, encontramos la referencia obligada a Basalla (1967), que aporta una concepción prescriptiva de la manera en que se mueve un concepto desde los "centros" de innovación hacia las "periferias". Es una sociología de la replicación que describe cómo los actores "adquieren" un conocimiento y lo promueven en sus contextos. Esta visión simplista de la realidad social y de los movimientos de la tecnociencia, ha sido duramente criticada por los teóricos de los ESCT que han visto en los modelos difusionistas como el de Basalla (1967), Pyenson (1993, 2002), Palladino y Worboys
(1993), un problema para comprender los movimientos de los conceptos, la apropiación y adaptabilidad de ellos, al igual que la implicación de los usos.

La crítica hecha desde los ESCT incluye la consideración de la movilidad como proceso que se construye en la interacción social con los dispositivos tecnocientíficos. Se ha planteado la importancia de indagar en cómo se han definido los hechos científicos como "verdad" y "certeza" (Latour \& Wolgar, 1979), la influencia de los contextos de apropiación en términos de los usos y los intereses personales y grupales (Chambers, 1993), la adaptación y performatividad de los usos (Jasanoff, 2004; Winner, 1993), sobre la relación entre expertos y no-expertos en el debate de la utilización, la recursividad del dispositivo tecno-científico en lo social y sus implicaciones en la construcción de la realidad (Jasanoff, 2005; Mulkay, 1997; Pinch, 1993; Winner, 1993; Wynne, 2004).

Lo que se logra rescatar a través de la indagación y la reflexión sobre la sospecha del modelo difusionista, es una perspectiva ampliada de las narrativas que son invisibilizadas en la construcción de los hechos considerados históricos. Además, un importante aporte a la posibilidad de observar esas otras versiones, es la posibilidad analítica que se deriva de una juiciosa atención a la red de actores que configuran los espacios de movimiento sobre el desplazamiento de un dispositivo tecnocientífico (Callon, 1986, 1987; Latour, 1991, 1992, 2008; Latour \& Wolgar, 1979).

Lo interesante de esta posibilidad analítica, es la ventaja procedimental. Esto debido a que produce una apertura de espacios entre las vetas historiográficas que permite observar la aparición de actores "no relevantes", los escenarios de construcción social, la traducción de interés y motivaciones personales e institucionales o la recurrencia de los "artefactos" como performadores de realidad. Sumado se encuentran las experiencias derivadas de los usos y las condiciones de reflexividad sobre las misma red como nodos de ocurrencia que producen sistemas sociológicos de acción (Callon, 1986, 1987; Law, 1991, 2004; Law \& Bijker, 1992; Law, Callon \& Rip, 1986).

Desde las anteriores perspectivas de trabajo que se han abierto paso en el escenario de los ESCT, existe una consideración importante sobre la relación entre dispositivos sociotécnicos presentes en escenarios como la construcción de la ciencia, la infraestructura, la tecnología, etc. Tomando como referente estas posibilidades analíticas, observaremos como se puede plantear una posibilidad historiográfica sobre la historia de la psicología y la pedagogía. 
Una relación explorada en los trabajos ya referentes sobre historia social de la ciencia, es la posibilidad de observar la movilidad de los dispositivos tecnocientíficos: conceptos, usos, estándares, etc. Esto en relación a las maneras de enseñanza y estilos de apropiación en el seno de comunidades y grupos de interés que tratan de adaptar los sistemas de conocimiento en términos pedagógicos para su reproducción contextual. Por supuesto, como se ha expuesto más arriba, la idea de movilización en términos de difusión deja brechas sobre la comprensión de la construcción del hecho social. Por otro lado, los trabajos que se concentran en dilucidar cómo es que el proceso de apropiación implica un suceso de traducciones de interés, sumado a unos pasos obligados y a procesos de adaptación, permiten clarificar las maneras en que se construyen los procesos de realidad en función de las operaciones sociotécnicas.

\section{Kaiser (2005) afirma:}

Pedagogy has long been a major concern of modern scientific professions. Hundreds of millions of dollars were spent on science pedagogy and its reforms throught out the nineteenth and twentieth centuries, both in the United States and in Europe. Whether focused on the introduction of laboratory techniques for a teaching in the late nineteenth century, on the highly touted multimedia productions of the physical Science Study Committee (PSSC), the Biological Science Curriculum Study (BSCS), and "Project Physics" during the Cold War, or on more recent efforts to incorporate computer simulations and more direct question-and-answer feedback into large introductory lectures, pedagogy has hardly been taken for granted by scientist themselves. (p. 1)

En la anterior cita, se observa la importancia de estudiar los escenarios pedagógicos que configuran los tipos de enseñanza sobre la física y la química. A su vez, anuncia una perspectiva interesante en términos metodológicos que ayuda a clarificar cómo es que la ciencia se mueve en contextos, vincula prácticas y artefactos y genera grupos de interés.

Un interesante ejemplo de lo anteriormente dicho es expuesto por Warwick (2003), pues en su trabajo permite observar cómo la consolidación de la física matemática en el Cambridge de la época Victoriana, tiene que ver con diferentes elementos: la escritura de un libro de texto en el que se canoniza el conocimiento que se debe impartir (Roth's Textbook); unas lecciones privadas a los estudiantes que suponen una interacción directa con un orientador, y el tratamiento del cuerpo como lugar donde se recepciona y alberga el conocimiento.

Siguiendo a Warwick (2003), se puede observar el reconocido caso de Félix Klein, matemático alemán, visitante recurrente de Cambridge y seguidor del método propuesto allí para la enseñanza de la matemática y la física. Klein pretende trasladar el método a Alemania, sin embargo, a la hora de la construcción de la metodología de aplicación, se encuentra con elementos contextuales que dificultan la labor: a. el lenguaje y su traducción al alemán; b. la imposibilidad de la aplicación de una tutoría individual por no comprender las acciones o los elementos que la componían.

Lo anterior pone de nuevo el énfasis en el lugar de la localidad y el significado de la apropiación de los discursos y prácticas en los escenarios locales. De Greiff y Maldonado (2011) han logrado mostrar cómo la apropiación del conocimiento refiere todo un análisis de las experiencias de interacción entre los movilizadores, los públicos y los escenarios de utilización de los artefactos y conceptos movilizados.

Es interesante mostrar también, cómo desde la interpretación de los planteamientos de Shapin (1984, 1994), se identifica una naturalización de las relaciones que emergen desde el experto y el lego, donde se instauran sujeciones y hasta sometimientos por el uso de una determinada taxonomía y una experticia técnica representada en el texto científico o artículo. En este sentido, los públicos se crean, las comunidades de recepción se constituyen y los hombres de ciencia, por supuesto, se construyen en la interacción resultante de los actores. De este modo, queda configurado todo un escenario para la comprensión de la movilización de elementos de un lugar a otro, y las pautas que un análisis de este tipo requieren para realizar un trazado de los elementos que configuran la red de movilización.

La revisión anterior destaca unas vías interesantes de trabajo que posibilitan la génesis de historias que visibilicen los fenómenos de co-producción de discursos locales, no-expertos. También ofrecen la posibilidad de rastrear la performatividad de los dispositivos en el escenario de los usos sociales. 


\section{El Gimnasio Moderno de Bogotá ${ }^{3}$ y la movilización de conceptos psicológicos y pedagógicos}

El desarrollo de la conceptualización sobre movilización y el apoyo de los ESCT han servido para encontrar vías de análisis de la historia tecnocientífica y social de la psicología y la pedagogía. Es evidente la relación estrecha que existe entre la puesta en escena social de los dispositivos conceptuales y prácticos derivados de las dos disciplinas. En el caso que se pretende presentar ahora, realizaremos una sucinta revisión del cruce entre lo psicológico y lo pedagógico en términos de una institución que ha sido el centro de enseñanza de la élite política de Colombia en un momento histórico particular 1915-1920. Se retoma este periodo de tiempo por su importancia en términos de la adaptación. Según trabajos de referencia, en estos años se observan procesos de recepción de los discursos sobre psicopedagogía con fuerte influencia de académicos que logran puentes con escuelas europeas, así como la adecuación de la infraestructura necesaria para la puesta en escena y la consolidación del método. Lo que sigue después puede ser considerado como un periodo de estabilización de los dispositivos sociotécnicos (Cubillos, 2007; Soto, 2015).

$\mathrm{Al}$ revisar las historias ligadas a la experiencia del Gimnasio Moderno de Bogotá (Cubillos, 2007; Helg, 1987; Sáenz et al., 1997), se puede definir una serie de actores importantes que permiten la proyección de conceptos y prácticas derivadas de la movilización de conceptos desde la psicología y pedagogía europea. Entre ellos se encuentran los actores institucionales, los estudiantes, los conceptos y recursos técnicos. Todos estos derivan en una sucesión de traducciones que construyen una realidad particular en el seno de la pedagogía activa y su aplicación en el centro educativo.

En primera instancia, se puede observar la importancia de personajes de relevancia en la primera mitad del siglo XX. Tal es el caso de Agustín Nieto Caballero, quien es considerado el precursor de la institución y la máxima autoridad en el tema de la "Escuela Activa". Es conocido por las historias precedentes de Sáenz (1990), Sáenz y Zuluaga (2004) y Cubillos (2007), que Agustín Nieto es el nodo que enlaza los conceptos de

\footnotetext{
Esta institución es un centro educativo ubicado en la Ciudad de Bogotá, fundado en 1914 con una orientación basada en la filosofía liberal, con criterios de la pedagogía de la Escuela Activa. Es reconocido por formar algunos de los dirigentes políticos e intelectuales más importantes en la historia del país.
}

la psicopedagogía europea en la Bogotá de los inicios del siglo XX.

Don Agustín, como es llamado, se alía con otros personajes estratégicos de la llamada "generación del centenario", algunos de ellos son José María Samper, Tomas Rueda Vargas y Alberto Lleras Codazzi, entre otros. Estos personajes tienen en común, una concepción de la educación como espacio de cambio y debate, en función de las ideas más tradicionales expresadas en los gobiernos conservadores de la época de la "Regeneración". Todos estos personajes tienen un aspecto que los identifica y este es la producción intelectual y su intervención en el campo político de la vida del país.

La reunión de estos personajes de la intelectualidad produce un proyecto educativo denominado Gimnasio Moderno, que se encargaría de reproducir los elementos de la filosofía liberal pregonada por sus fundadores y, en virtud de ella, debería ostentar una epistemología y metodología que reflejara los principios liberales en la constitución de sujetos potencialmente aptos para dirigir una reforma modernista en el país (Nieto, 1935).

$\mathrm{Y}$ es aquí donde tiene un posible punto de partida la movilización de conceptos de la psicología europea. Los contactos e influencias de Agustín Nieto llevan a considerar la posibilidad de fundar una institución que proyecte la novedosa forma de entender el mundo individual de la psicología europea representada en dos autores, María Montessori (1937) y Ovide Decroly (1932). Los contactos del autor son el elemento vinculante entre la Bogotá de 1915, la sociedad, sus estudiantes, la ideología liberal y la consigna por una educación que cambie al sujeto pasivo conservador en un sujeto activo liberal.

Los informes del Gimnasio Moderno en la época (1915-1920) dejan ver cómo se organiza la puesta en escena de la apropiación social del conocimiento específico a partir de la psicopedagogía de la escuela europea. Se observa una delimitación del infante como centro de recepción de los usos y estrategias prácticas de la conceptualización psicológica y pedagógica. El estudiante es un actor que concentra en sí mismo la renovación de la pedagogía, es uno "paso obligado" de los recursos conceptuales y técnicos que se implantan

\footnotetext{
Se conoce como "Generación del Centenario" a un grupo de intelectuales con vocación política liberal que introducen, desde varios escenarios de la vida pública del país, una serie de debates sobre la concepción de la orientación política conservadora que desde finales del siglo XIX ostentaba el poder y la dirección del país.
} 
en función de la renovación educativa que se proclama en la institución.

Lo llamó "paso obligado", en el sentido atribuido por Callon (1986). En la red de actores de este, hay un nodo que concentra la traducción de intereses y es el reflejo de la movilidad. Este nodo como actor, concentra el foco de atención y es agente productor de significado en el que convergen distintas interacciones. Ya en "Mirar la infancia" se observa una designación importante del concepto de infancia como centro del control en el que recae la vigilancia y el control del cuerpo y del sí mismo, así como una propiedad escalar que incluso concentra en el niño la posibilidad de construcción de una moral e intelectualidad liberal que propende por un desarrollo moderno (Sáenz et al., 1997).

Con esta referencia sobre la infancia como objeto, se abre el debate, y se pueden generar formas alternativas de análisis. En este caso, es posible tomar el dispositivo como un elemento vinculante, como un "paso obligado" en el que se observan diferentes conexiones con otros nodos de la red que además producen una cierta construcción de la realidad social de la educación. Así las cosas, el concepto de infancia ya no se puede pensar como una referencia esencial, sino como una intersección de intereses, mediaciones, traducciones, etc.

Ahora bien, en este sentido los dispositivos conceptuales de las teorías psicológicas y pedagógicas, definen un problema particular sobre la infancia, se refieren a la mejor forma de entender el proceso de desarrollo y cómo hacer para potenciar la actividad intelectual y cognitiva. Así pues, se va ordenando una red de actores que movilizan las conceptualizaciones, tenemos a Agustín Nieto, el Gimnasio Moderno, la infancia representada en los estudiantes, y por supuesto los nodos que en Europa se tornan aspecto importante, pues de ese "centro" de producción se mueven conceptos y prácticas que tienen un interés por la difusión. Hablamos entonces de conceptos que se generan en el contexto de la Escuela de Ginebra, una psicología experimental y estructuralista que se abre paso entre las nacientes perspectivas para explicar el proceso de mediación entre la capacidad de conocimiento y el desarrollo evolutivo de la filogenia y la ontogenia.

Los dos representantes de importancia en los inicios de esta historia, son María Montessori y Ovide Decroly, quienes formularon conceptualizaciones sobre un infante activo que desarrolla su potencial de aprendizaje en el contacto directo con los objetos del mundo, afirmando un desarrollo por niveles o estadios que serán la base de la psicología desarrollista y de la pedagogía activa. Cada uno de estos autores tiene un espacio de afirmación importante en sus contextos, vemos la "Casa dei Bambini" de María Montessori en Italia y el Centro de Enseñanza para niños con limitaciones cognitivas de Decroly en Bélgica. Agustín Nieto tiene la oportunidad de conocer a los autores en sus viajes a Europa y en su estancia Universitaria en Francia, y decide que el concepto de desarrollo y observación experimental se liga muy bien con el deseo de una filosofía liberal que proyecte un tipo innovador de educación (Decroly, 1932) .

Es así como se proyecta una elaboración en red para movilizar los conceptos desde la Europa de inicios de siglo, a la Bogotá de gobierno conservador. Se observa entonces la disposición de Agustín Nieto para llevar a cabo el proyecto de dirigir un espacio institucional que aporte los elementos necesarios para la innovación de la educación, y así se lleva a cabo el proyecto de la escuela activa, partiendo de las consideraciones de la teoría del desarrollo: "En la misma Europa, en donde imaginamos que todo ha de ser perfecto, esta reforma está apenas iniciada; estudiemos los problemas que plantea, y sin detenernos a atacar los puntos vulnerables, consideremos todo lo que fácilmente podríamos adaptar" (Nieto, 1917, p. 21).

Ahora bien, si se quiere avanzar en un análisis de la movilización, se debe observar también la localidad como elemento que interactúa con las condiciones de movilidad y adaptabilidad. Hemos visto en los trabajos de Restrepo (1998, 2000), Nieto (2006), De Greiff y Nieto (2005) y Mora (2012), cómo los hechos de movilización de un sistema teórico, de una artefacto o de una práctica discursiva, son performados por las características contextuales y resultan en formas de adaptación de los conceptos y prácticas de tal manera que no son calculables o concebidos en la misma expectativa de la actividad de difusión.

En el escenario de la iniciativa de psicología y pedagogía activa en el Gimnasio Moderno, se observan características interesantes en el proceso de apropiación. Tal es el caso de la práctica de asignación de cursos que se ejecuta realizando un examen de las características cognitivas en función de la edad, tal como supone la teoría de Montessori y Decroly:

\footnotetext{
5 Se recomienda el texto de la visita de Ovide Decroly a Colombia, específicamente su referencia a la comunicación fluida con Agustín Nieto y el interés que despierta la adaptación de sus métodos en el Gimnasio Moderno.

6 La negrilla es mía.
} 
Nuestro Inspector Médico, Doctor don José María Montoya, ha comenzado las mediciones antropométricas escolares de nuestros alumnos (...) con experimentos psicológicos, para poder establecer la ficha fisiológica, psicológica y escolar de cada uno de nuestros alumnos, como base para un conocimiento más completo de su personalidad total. (Nieto, 1917, p. 69)

La anterior cita muestra un "móvil inmutable", una categoría latouriana para definir los elementos no variables en un escenario de movilización (Latour, 2008). En este caso el aspecto de la evaluación del desarrollo y la aplicación, son derivados de los estudios de los autores europeos y nada tienen de diferencia a las consideraciones de aplicación. Si se observan los trabajos de Decroly y Monchamp (1932) o Montessori (1937), y se realiza un paralelo con los procedimientos aplicados en la evaluación de las características, capacidad intelectual y estadio del desarrollo, en el gimnasio moderno (Nieto, 1917), se puede observar una reproducción fiel de la metodología de evaluación y aplicación de test.

Las diferencias comienzan a interactuar contextualmente, en función de las formas de aplicación de los procesos de enseñanza de los contenidos instruccionales y comportamentales. Tal es el caso de la diferenciación que se hace de las formas de educar, una forma instruccionista y otra educacional que está dirigida a controlar el comportamiento de los estudiantes: "La base de lo que nosotros hemos llamado estudios de la realidad es el Plan de Estudios de las Escuelas del Doctor Decroly, de Bruselas, con algunas modificaciones para adaptar dicho Plan a Colombia" (Nieto, 1917, p. 117).

De este modo, se puede ver la situación del móvil en la red, se ha ubicado en un nodo en el que los conceptos necesitan ser contextualizados y adaptados, en este caso observaremos tres formas de adaptación que derivan de las demandas contextuales al método: 1. El control del cuerpo y la sexualidad como disciplina y autocontrol; 2. La religión como demanda de una educación moral sustentada en los preceptos católicos, y 3. El Gobierno Escolar como proyección de la política liberal de autoridad (Nieto, 1917).

La idea de un cuerpo que se debe controlar se expresa en la necesidad de ocupar la mente del niño con el trabajo intelectual y la disciplina como instrumento para tal fin. Lo que ocurre, en función de una apropiada disciplina, es la utilización de la instrucción sobre el cuerpo a través de la gimnasia. Los cursos en la institución son combinados con momentos de gimnasia y educación física, donde los niños son puestos en actividades de trabajo físico con el fin de desarrollar un proceso de formalización de su estado corporal. Además, a través de este mecanismo, se observan discursos asociados a que la disciplina corporal, fomenta la regulación del comportamiento sexual, un problema que suscita varias formas de tratamiento: "Se planteó la cuestión en esta forma: «¿Cómo podríamos inmunizar el alma y el cuerpo del niño de las influencias perniciosas de la sexualidad?»" (Nieto, 1917, p. 40).

Lo que se plantea es la necesidad de control sobre el cuerpo y, por ende, sobre lo que se considera como "alma" o "mente"; se necesita un dispositivo que regule la conducta, para ello la gimnasia, combinada con una serie de charlas sobre el tema de la sexualidad como una actividad inmoral, que no debe ser preocupación de los niños, son formas de vigilancia y autocontrol sobre el cuerpo.

Una segunda variedad del método, es la inclusión de la actividad de enseñanza de la religión católica. En este sentido, la institución recoge los servicios de un presbítero y da un espacio de cátedra semanal a cada curso para recibir instrucción sobre el dogma y las enseñanzas morales del catolicismo. Dado que se encuentra una demanda de parte de los padres de familia sobre la enseñanza de la religión católica, el Gimnasio y sus directivas a la cabeza, deciden poner en funcionamiento la cátedra de religión. Ésta ayudará en el proceso de justificación de una moral prescriptiva, que ayuda para fundamentar la decisión sobre el autocontrol del comportamiento corporal como correlato de una vida mental y espiritual sana:

El siguiente es el plan que combina la disciplina corporal a través del ejercicio, el entretenimiento y la religión, además de la vigilancia y permanente auditoría de los maestros:

1. Procurar unificar la acción educativa de confesores, padres y maestros para evitar las influencias de la sexualidad del niño.

2. Aprovechar un momento ocasional para hablar a los muchachos precoces de una manera austera y paternal del misterio de la procreación, del amor de la madre, del respeto a la mujer y de los males del vicio.

3. En los estudios de ciencias naturales los profesores hablarán, con fines educativos y científicos a la vez, de la reproducción de animales y plantas.

4. En las lecciones de historia y arte se hará sentir a los niños la serenidad de la belleza, mostrándoles, cuando la cuestión se tercie, el desnudo en la estatuaria griega o en la pintura de los grandes maestros, para 
que, conociendo lo bello, les repugne lo sensual y pornográfico, puesto que lleva en sí el estigma de la fealdad.

5. Ocupar intensamente la imaginación de nuestros alumnos con trabajos y estudios, y su cuerpo con juegos fatigantes para que no les dé ocasión ni ganas de entregarse a concepciones y prácticas deshonestas.

6. Avivarles sobre todo el santo temor de Dios y estimularles a que acudan a Él para hallar fortaleza en sus debilidades por medio de los sacramentos repetidos con frecuencia (Nieto, 1917, p. 41)

Por último, se debe hablar de una de las estrategias más interesantes para la promoción de los intereses liberales y modernistas de la institución, la "República Escolar". En esta forma adaptada de un gobierno de Estado, el Gimnasio aborda el problema del comportamiento y la moral del infante sobre el precepto de autoridad representativa. Esto quiere decir que los estudiantes elegirán, entre candidatos idóneos de su propio curso, un representante como figura de autoridad y de interlocución con profesores y directivas.

De este modo, los estudiantes asumen la autoridad de señalar a aquellos que han cumplido con los preceptos morales y aquellos que no, de esta manera se forman consejos de estudiantes representantes, profesores y directivas para formular casos de estudiantes que necesitan un encauzamiento de su comportamiento, lo que sirve a la institución para acortar la posible parcialidad por parte de juzgamientos unilaterales de profesores a estudiantes.

El sistema de una "República Escolar" distribuye la autoridad y entrega parte de ella a los estudiantes, aunque con ello también la responsabilidad de vigilancia del comportamiento de los miembros de su propio grupo, lo que genera una actividad de vigilancia permanente que puede ser aprovechada por la institución para controlar de manera menos explícita a los infantes; es así como se efectúa un proceso de adaptación en función de la localidad. Como hemos visto, el control del comportamiento se deriva de las demandas funcionales de la infancia como objeto de una moral religiosa y conservadora que se renueva en el ejercicio de aplicación de un sistema pedagógico de corte activo. En este, el sujeto puede pertenecer a un gobierno escolar, se ve implicado en formas de autocontrol, en una vigilancia entre pares y multinivel, y en la construcción de una red que es movida y operada por los conceptos tecnocientíficos.
Ahora bien, se observó cómo desde una perspectiva de apropiación, se puede pasar a una forma de análisis de la red de actores a través de la cual se van moviendo los conceptos, las prácticas, y de la que van emergiendo necesidades contextuales que se suplen a partir de las innovaciones en los métodos y prácticas discursivas. El caso de la educación en el Gimnasio Moderno, es un ejemplo interesante de la amplitud analítica de los estudios sociales puestos al servicio de la investigación historiográfica de la psicología y la pedagogía.

\section{Discusión}

\section{Sobre las posibilidades analíticas desde los ESCT en la historiografía de la psicología y pedagogía}

Es importante reconocer que la historia de la psicología, como lo han planteado varios estudios que han utilizado los ESCT como herramienta epistemológica y metodológica, ha resultado ser provechosa en sus variantes analíticas, pues han concentrado sus esfuerzos en generar una descripción de las formas en que los dispositivos tecnocientíficos se mueven por escenarios sociales con una clara forma contextualizada de acción.

Se puede notar entonces que el seguimiento de un determinado corpus teórico se relaciona con las formas contextuales en las que se mueve; se ha visto cómo el dispositivo conceptual y práctico de la psicología y pedagogía activa, se mueve por escenarios que van desde un aparente "centro" de constitución, hasta un destino poco sospechado como la Bogotá de inicios de siglo $\mathrm{XX}$, donde se ubica en un escenario político, religioso y social particular que demanda unas modificaciones para su apropiación y utilización. Es importante analizar que existen precedentes que vinculan esa tradición de la movilización de conceptos y métodos de una escuela a otra, un claro ejemplo es el plan de estudios sobre matemáticas de Cambridge que se quiere apropiar en Alemania. En este caso las dificultades de traducción de los textos, la metodización de la disciplina corporal y las características de los estudiantes en Alemania, dificultan y generan una serie de adaptaciones del método que resultarán en formas diversas de comprenderse y aplicarse (Olesko, 2005; Warwick, 2003).

Estas referencias ponen de manifiesto la necesidad de encontrar vías alternas a las ya enunciadas en los modelos difusionistas de Basalla (1967) o McLeod (1980) que intentan descripciones de los movimientos 
sin una profunda comprensión de la movilización como condición emergente de nuevas lógicas conceptuales y prácticas de los usos de la tecnociencia. Como lo ha mostrado Restrepo $(1998,2000)$, los efectos de la movilización de conceptos y artefactos, no sólo dejan ver la posibilidad de conformación de un tipo de uso innovador en el seno de un contexto social, sino que permite la apertura a novedosas lógicas de interacción entre pares y relaciones institucionales. Estas últimas, se originan o se modifican en función de la producción de conceptos, de lógicas interactivas de agencia social e incluso de perspectivas emergentes de formas relacionales y morales. Como hemos visto en el caso del Gimnasio, rastrear la teoría psicopedagógica nos permite comprender el devenir de ciertas formas de adaptación de lo que es considerado como un estudiante, de las formas en que ese estudiante es considerado un sujeto liberal y de lo que trae consigo esas consideraciones en el entorno formativo y educacional que demanda un cierto tipo de "ethos" en su constitución y en su proyección en la sociedad.

Es así como el escenario de los Estudios Sociales de la Ciencia abre una cantidad incontable de narraciones sobre las que se puede realizar historiografía de la psicología, atendiendo a eso que Vezzetti (1996, 2007), Kapplenbach y Pavessi (1994), Jaraba (2012) y otros tantos, han sugerido como un replanteamiento de la manera de atender la historia y sus implicaciones en las múltiples construcciones de la psicología como ciencia, como disciplina, como artefacto, como discurso, etc. Los ESC pueden brindar las herramientas metodológicas para acercarnos a un diálogo con las narrativas y los dispositivos que se han configurado en el recorrido histórico de las diversas psicologías, utilizando el recurso del seguimiento en red (Moreno, 2014), de la revisión y análisis de controversias (Jaraba, 2012; Mora, 2012), trabajos sobre apropiación social y campos de experticia (Pulido-Martínez, 2006, 2011).

Estás formas de realizar historiografía representan un trabajo enriquecedor y abren diversas vertientes de análisis sobre problemáticas que en las versiones más tradicionales de la historia de la psicología en Colombia, se han dejado olvidadas en una suerte de "caja negra". Es una oposición analítica a la tradicional historia celebratoria que con una retórica difusionista conduce a los interesados por la historia de la psicología a recorrer un solo camino, con una única verdad privilegiada que resta importancia a tantas narrativas interesantes e importantes para comprender el devenir histórico de la diversidad de las psicologías en nuestro país.
De acuerdo a lo anterior, se ofrece la posibilidad de estudiar en red la importancia de los actores como nodos y como agentes de construcciones de la realidad. En este caso se ha seleccionado a un actor importante para definir un punto de inicio en el ejercicio de mapear la red y seguir los dispositivos conceptuales y prácticos, es el caso de Agustín Nieto. Ya en trabajos de referencia, se ha utilizado la figura del actor como nodo y punto de partida para el análisis de las redes y las construcciones de la realidad que emergen en la interacción con otros actores y actantes, tal es el caso de Joliot y la física de partículas en Francia (Latour, 1991) o Pasteur y la definición de los microorganismos (Latour, 1988).

En el caso de Agustín Nieto, hemos visto cómo se puede localizar un nodo y observar, a partir de él, unas condiciones de negociación en relación a una red específica. En este sentido, se ha observado el lugar de agente traductor de intereses políticos, tecnocientíficos y sociales que posibilita el escenario de la educación. La realidad se ve modificada y tramitada por los efectos de adaptación y aplicación de dispositivos psicopedagógi$\cos , \mathrm{y}$ emergen instituciones y agentes que respaldan el accionar, y que a la postre, se involucran como actores relevantes en el contexto de la red.

La elección de un punto de partida en la observación, de un actor relevante, no es una recaída en la "historia heroica" o "épica" que visibiliza a un agente difusionista para que recaiga sobre él todo el crédito de la movilización. Contrario a esta percepción, se refiere a la posibilidad de concentrar el punto de inicio para rastrear los movimientos que emergen de la red, los nodos que se ligan y las relaciones que se derivan. Es una estrategia para dar inicio y dirección al objetivo de rastreo de las posibilidades analíticas, no para dar un centro jerárquico de importancia, sino para construir a partir de un punto, el más visible en ocasiones, la búsqueda y exploración de alternativas.

En este texto se quiso realizar un ejercicio de localización de un actor para mostrar cómo a partir de él se puede mover el mapeo de la red y construir las relaciones para que emerjan las categorías a analizar; se produjo un contraste, para que aquellos que han leído y conocen la historia de la psicología en Colombia, o los que estén interesados en conocerla, puedan observar cómo en esas historias canónicas y "épicas", el seguimiento de la red es limitado y se advierte una dinámica de análisis difusionista que para nada permite el encuentro con los contextos y las relaciones entre nodos, intereses y motivaciones que son los que ayudan 
a comprender la construcción de diferentes realidades sociales.

\section{Referencias}

Ardila, R. (1973). La Psicología en Colombia, Desarrollo Histórico. Trillas. México.

Ardila, R. (1975). La Historia de la Psicología en Colombia y el Plan Quinquenal 1970-1975. Revista Latinoamericana de Psicología, 7(3), 435-446. Recuperado de http://www.redalyc.org/articulo. oa? $\mathrm{id}=80570308$

Ardila, R. (1999). Las Ideas psicológicas en Colombia. Revista de Estudios Sociales, (3), 59-67. Recuperado de http://res.uniandes. edu.co/view.php/82/index.php?id=82

Ardila, R. (2004). La Psicología Latinoamericana: El Primer Medio Siglo. Revista Interamericana de Psicología, 38(2), 317-322. Recuperado de http://www.psicorip.org/Resumos/PerP/RIP/ RIP036a0/RIP03834.pdf

Ardila, R. \& Pérez-Acosta, A. (2010). La Psicología Colombiana: Revistas y Bibliografía. Revista Latinoamericana de Psicología, 42(3), 517-523. Recuperado de http://www.redalyc.org/articulo. oa? $\mathrm{id}=80515851019$

Basalla, G. (1967). The Spread of Western Science. Science, 156(3775), 611-622. doi: 10.1126/science.156.3775.611

Callon, M. (1986). Some Elements of a Sociology of Translation: Domestication of the Scallops and the Fishermen of St Brieuc Bay. En J. Law (Ed.), Power, Action \& Belief: A New Sociology of Knowledge? (pp. 196-229). London: Routledge.

Callon, M. (1987). Society in the Making: The Study of Technology as a Tool for Sociological Analysis. En W. Bijker, T. Hughes \& T. Pinch (Eds.), The Social Construction of Technical Systems: New Directions in the Sociology and History of Technology (pp. 83-103). Cambridge: MIT Press.

Chambers, D. (1993). Localidad y Ciencia Mitos de Centro y Periferia. En A. Lafuente, A. Elena \& M. Ortega (Eds.), Mundialización de la ciencia y cultura nacional: actas del Congreso Internacional Ciencia, Descubrimiento y Mundo Colonial (pp. 605-617). Madrid: Doce Calles.

Cubillos, J. (2007). Agustín Nieto Caballero y el Proceso de Apropiación del Pensamiento Pedagógico y Filosófico de Jhon Dewey. Cali: Universidad del Valle.

Decroly, O. (1932). El Doctor Decroly en Colombia. Bogotá: Imprenta Nacional.

Decroly, O. \& Monchamp, E. (1932). La iniciación a la Actividad Intelectual y Motriz por los Juegos educativos. Madrid: Imprenta Torrent.

De Greiff, A. \& Maldonado, O. (2011). "Apropiación Fuerte" del conocimiento: una propuesta para construir políticas inclusivas de ciencia, tecnología e innovación en América Latina. En A. Arellano \& P. Kreimer (Dir.), Estudio Social de la Ciencia desde América Latina (pp. 209-262). Bogotá: Siglo del Hombre Editores.

De Greiff, A. \& Nieto, M. (2005). Anotaciones para una agenda de investigación sobre las relaciones tecnocientíficas Sur-Norte. Revista Estudios Sociales, 22, 59-69. Recuperado de http://www. scielo.org.co/scielo.php?script=sci_arttext\&pid=S0123-885X200 $5000300005 \& \operatorname{lng}=$ en\&nrm=iso\&tlng=es

Gutierrez, G., Pérez-Acosta, A. \& Plata-Caviedes, T. (2009). Desarrollo Histórico de una Publicación Científica: Cuarenta Años de la Revista Latinoamericana de Psicología. Revista Latinoamericana de Psicología, 41(3), 413-442. Recuperado de http://www.redalyc. org/articulo.oa?id=80511929003
Helg, A. (1987). La Educación en Colombia 1918-1957: Una Historia Social, Económica y Política. Bogotá: CEREC.

Jaraba, B. (2012). Los "orígenes" de la psicología colombiana: entre la épica y el archivo. Cuadernos de Psicología, 8(2), 37-68.

Jasanoff, S. (2004). The States of Knowledge: The Co-Production of Science and Social order. London: Routledge.

Jasanoff, S. (2005). Designs of Nature: Science and Democracy in Europe and the United States. Princeton: Princeton University Press.

Kaiser, D. (2005). Introduction: Moving Pedagogy from the Periphery to the Center. En D. Kaiser (Ed.), Pedagogy and the Practice Of Science. Historical and Contemporary Perspectives (pp. 1-8). Cambridge: MIT Press.

Klappenbach, H. \& Pavessi, P. (1994). Una Historia de la Psicología en América Latina. Revista Latinoamericana de Psicología, 26(3), 445-481. Recuperado de http://www.redalyc.org/articulo. oa? id $=80526305$

Latour, B. (1988). The Pasteurization of France. Cambridge: Harvard University Press.

Latour, B. (1991). Joliot: punto de encuentro de la historia y de la física. En M. Serres (Coord.), Historia de las Ciencias (pp. 553573). Madrid: Ediciones Cátedra.

Latour, B. (1992). Ciencia en acción: cómo seguir a los científicos e ingenieros a través de la sociedad. Barcelona: Labor.

Latour, B. (2008). Reensamblar lo social. Una introducción a la teoría del actor-red. Buenos Aires: Ediciones Manatial.

Latour, B. \& Woolgar, S. (1979). The Life in the Laboratory. Princeton: Princeton University Press.

Law, J. (1991). A Sociology of monsters: essays on power, technology, and domination. London: Routledge.

Law, J. (2004). After method: mess in social science research. London: Routledge.

Law, J. \& Bijker, W. (1992). Shaping technology/building society: studies in sociotechnical change. Cambridge: MIT Press.

Law, J., Callon, M. \& Rip, A. (1986). Mapping the dynamics of science and technology: sociology of science in the real world. Basingstoke: Macmillan.

López, W. (1997). La Investigación En la Psicología Colombiana: 50 años de pasado, un milenio de retos y futuro. Boletín de la Sociedad Colombiana de Psicología, (16), 11-12.

MacLeod, R. (1980). On Visiting the 'Moving Metropolis': Reflections on the Architecture of Imperial Science. Historical Records of Australian Science, 5(3), 1-16. doi:10.1071/HR9820530001

Makeliunas, M. (1980). Desarrollo de la Psicología en Colombia y su ubicación como Ciencia. En G. Aldana \& M. González (Eds.), La Psicología ¿Ciencia Social? (pp. 19-33). Bogotá: Pontificia Universidad Javeriana.

Makeliunas, M. (1993). Desarrollo Histórico. En R. Ardila (Comp.), Psicología en Colombia Contexto Social e Histórico (pp. 43-66). Bogotá: Tercer Mundo.

Montessori, M. (1937). El Método de la Pedagogía Científica aplicada en la Casa de los Niños. Barcelona: Araluce.

Mora, F. (2012). Colombian Psychology: Knowledge, Technique and Controversies. Reconstructions from Social Studies of Science (Tesis de maestría). Bogotá: Universidad Nacional de Colombia.

Moreno, P. (2014). Producción de evidencia psicológica en el debate jurídico sobre adopción gay en Colombia. Universitas Psychologica, 13(5), 1931-1940. doi:10.11144/Javeriana.upsy13-5.pepd

Mulkay, M. (1997). The Embryon Research Debate. Science and the Politics of Reproduction. New York: Cambridge University Press.

Nieto, A. (1917). Gimnasio Moderno. Informes relativos al curso de 1916. Bogotá: Editorial Arboleda \& Valencia.

Nieto, A. (1935). Labores de la Dirección Nacional de Educación. Bogotá: Editorial ABC. 
Nieto, M. (2006). Remedios para el Imperio: historia natural y la apropiación del nuevo mundo. Bogotá: Universidad de Los Andes.

Olesko, K. (2005). The Foundations of a Canon: Kohlrausch's Practical Physics. En D. Kaiser (Ed.), Pedagogy and the Practice Of Science Historical and Contemporary Perspectives (pp. 323-356). Cambridge: MIT Press.

Oviedo, G. (2004). La definición del Concepto de Percepción en Psicología con Base en la Teoría de la Gestalt. Revista de Estudios Sociales, (18), 89-96. Recuperado de http://res.uniandes.edu.co/ view.php/375/index.php?id=375

Oviedo, G. (2009). Concepciones Psicológicas Colombianas en el siglo XIX. Revista de Historia de la Psicología, 30(4), 7-32. Recuperado de http://dialnet.unirioja.es/servlet/articulo? codigo $=3130677$

Oviedo, G. (2010). Lecciones de Psicología-Colombia Siglo XIX. Avances de Psicología Latinoamericana, 28(2), 278-294. Recuperado de http://www.scielo.org.co/pdf/ap1/v28n2/v28n2a11.pdf

Oviedo, G. (2013). El Proceso Secular en de la Conciencia Psicológica en Colombia (1886-1917) (Tesis doctoral). Bogotá: Universidad Nacional de Colombia.

Palladino, P. \& Worboys, M. (1993). Science and Imperialism. Isis, 84(1), 91-102.

Peña, T. (1993). La psicología en Colombia: Historia de una Disciplina y una Profesión. En C. Vasco, D. Obregón \& L. Orozco (Coords.), Historia Social de la Ciencia en Colombia. Tomo IX: Ciencias Sociales (pp. 95-179). Bogotá: Colciencias.

Pérez-Acosta, A. (1997). La Psicología Colombiana: Un Adolescente de cincuenta años. Boletín de la Sociedad Colombiana de Psicología, 16, 1-3.

Pinch, T. (1993). La Retórica y la Controversia sobre la Fusión Fría: del Woodstock químico al Altamont Físico. Política y Sociedad, 14-15, 155-170. Recuperado de http://revistas.ucm.es/index.php/ POSO/article/view/POSO9394110155A/30360

Pulido-Martínez, H. (2006). Produciendo Trabajadores Modernos: Conocimiento Psicológico y el Mundo del Trabajo en el Sur. Universitas Psychologica, 6(1), 27-37. Recuperado de http:// revistas.javeriana.edu.co/index.php/revPsycho/article/view/91/16

Pulido-Martínez, H. (2011). Psicología y trabajo: una relación bajo examen. En B. P. Ballesteros de Valderrama \& H. Escobar Melo (Eds.), Psicología y políticas públicas: aportes desde la academia (pp. 123-144). Bogotá: Pontificia Universidad Javeriana.

Pyenson, L. (1993). The Ideology of Western Racionality: History of Science and the European Civilizing Mision. Science and Education 2, 329-343. doi:10.1007/BF00488170

Pyenson, L. (2002). Comparative History of Science. History of Science, 40(127), 1-33. Recuperado de http://riseofthewest.com/ dc/dc247pyenson00mar02.pdf
Restrepo, O. (1998). En busca del orden: ciencia y poder en Colombia. Asclepio, 50(2), 33-75. doi:10.3989/asclepio.1998.v50.i2.336

Restrepo, O. (2000). La sociología del conocimiento científico o de cómo huir de la "recepción" y salir de la "periferia". En D. Obregón (Ed.), Culturas científicas y saberes locales (197-220). Bogotá: Universidad Nacional de Colombia.

Sáenz, J. (1990) Psicología y Escuela Activa en Colombia. Pedagogía y Saberes, (1), 36-47. Recuperado de http://www.ascofapsi.org. co/documentos/2009/Psicologia_escuela_activa.pdf

Sáenz, J., Saldarriaga, O. \& Ospina, A. (1997). Mirar la Infancia. Pedagogía, Moral y Modernidad en Colombia 1903-1946. Bogotá: Colciencias.

Sáenz, J. \& Zuluaga, O. (2004). Las Relaciones entre Psicología y Pedagogía: Infancia y Prácticas de Examen. Memoria y Sociedad, 8(17), 9-26. Recuperado de http://www.javeriana.edu.co/ Facultades/C_Sociales/memoria/memoria17/psicologia.pdf

Shapin, S. (1984). Pump and Circumstance: Robert Boyle's Literary Technology. Social Studies of Science, 14(4), 481-520. Recuperado de http://www.fas.harvard.edu/ hsdept/bios/docs/ shapin-pump_circum.pdf

Shapin, S. (1994). A Social History of Truth: Civility and Science in Seventeenth-Century England. Chicago: The Chicago University Press.

Soto, J. (2015). Contextos de la psicología: Don Agustín Nieto Caballero, sus relaciones y la construcción de la pedagogía (Tesis de maestría inédita). Universidad Nacional de Colombia, Bogotá.

Vezzetti, H. (1996). Los estudios históricos de la psicología en la Argentina. Cuadernos Argentinos de Historia de la Psicología, 2(1-2), 79-93. Recuperado de http://elseminario.com.ar/biblioteca/ Vezzetti_estudios_historicos.htm

Vezzetti, H. (2007). Historias de la Psicología: Problemas, Funciones y Objetivos. Revista de Historia de la Psicología, 28(1), 147-166. Recuperado de http://dialnet.unirioja.es/servlet/ articulo? codigo $=2291020$

Warwick, A. (2003). Masters of Theory. Cambridge and the Rise of Mathematical Physics. Chicago: The University of Chicago Press.

Winner, L. (1983). Do artifacts have Politics? En D. MacKenzie \& J. Wajcman (Eds.), The Social Shaping of Technology (pp. 28-40). Philadelphia: Open University Press.

Wynne, B. (2004). ¿Pueden las Ovejas Pastar Seguras?: Una mirada reflexiva sobre la separación entre conocimiento experto - conocimiento lego. Revista Colombiana de Sociología, (23), 109-157. Recuperado de http://www.revistas.unal.edu.co/index.php/recs/ article/view/11274/11934 
